\title{
Face of the giant panda sign in Wilson disease
}

\author{
S Chakraborty, ${ }^{1}$ MB BS, MD (Radiodiagnosis); S Mondal, ${ }^{2} \mathrm{MB} B S, \mathrm{DMRD} ; \mathbf{D}$ Sinha, ${ }^{2} \mathrm{MB} B S ; \mathrm{A} \mathrm{Nag},{ }^{2} \mathrm{MB} B S$
}

${ }^{1}$ Department of Radiology and Imaging, Institute of Post Graduate Medical Education and Research, Kolkata, India

${ }^{2}$ Department of General Medicine, Institute of Post Graduate Medical Education and Research, Kolkata, India

Corresponding author: S Mondal (drmsumantro@gmail.com)

\begin{abstract}
Wilson disease usually presents with neurological or hepatic manifestations. Magnetic resonance imaging (MRI) of the brain is most informative in diagnosing this disease, especially in patients with neurological features. High T2 signal intensity in the corpus striatum is the most commonly encountered MRI finding. The 'face of the giant panda' sign, seen on axial T2-weighted MRI, results from abnormal signal intensities in the midbrain. Though uncommon, the sign is regarded as the pathognomonic MRI sign of Wilson disease.
\end{abstract}

S Afr J Rad 2013;17(3):104. DOI:10.7196/SAJR.855

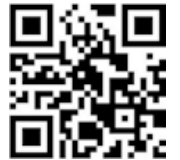

An 18-year-old man was admitted with a gradually progressive tremor and abnormal movements of the limbs of 6 months' duration.

There was no evidence of sensory or pyramidal tract involvement. He had no past or family history of liver disease. Examination revealed a coarse appendicular tremor $(5-6 \mathrm{~Hz})$ and choreiform movements of the hands, along with mild hepatomegaly. Wilson disease (WD) was confirmed by documentation of Kayser-Fleischer rings in both eyes, a low serum ceruloplasmin level $(15 \mathrm{mg} / \mathrm{dl})$ and increased 24-hour urinary copper excretion (430 $\mu \mathrm{g} / 24 \mathrm{hr}$ ). Axial T2-weighted MRI of the brain showed the characteristic 'face of the giant panda' sign in the midbrain (Fig. 1) and hyperintensity in the basal ganglia and thalamic regions bilaterally (Fig. 2).

\section{Discussion}

MRI of the brain is not only a useful diagnostic modality in WD but can also be used to assess disease severity and response to treatment. MRI changes are seen in virtually all neurologically symptomatic patients. ${ }^{[1]}$ When patients have predominant hepatic involvement, T1 hyperintensity is noted in the globus pallidus, putamen and mesencephalon. In neurologically symptomatic patients, T2 hyperintensity is noted in the putamina, caudate nuclei, thalami, midbrain and pons. Atrophy of the cerebrum and brainstem may be seen in long-standing cases. ${ }^{[1,2]}$ The 'face of the giant panda' sign, seen on axial
T2-weighted images of the midbrain, is regarded as characteristic of WD. ${ }^{[3]}$ The sign was originally described by Hitoshi et al.; it is produced as a result of high signal intensity in the tegmentum with preserved normal signal intensity in the red nuclei (eyes of the panda) and lateral portion of the pars reticulata of the substantia nigra (ears of the panda), and hypointensity of the superior colliculi (chin of the panda). ${ }^{[3]}$

The exact pathogenesis of the superior colliculus hypointensity is not known. It has been postulated that the paramagnetic effect of heavy metal deposition (e.g. iron, copper) may be responsible for this finding. ${ }^{[4]}$ In $\mathrm{WD}$, iron deposition is more significant than copper for producing T2 hypointensity. The 'face of the giant panda' sign is not commonly encountered. In a study of 100 patients, it was present in $12 \% .^{[1]}$ However, the sign is pathognomonic of WD, being the only MRI feature that distinguishes WD from other early onset extrapyramidal disorders. ${ }^{[5]}$

1. Sinha S, Taly AB, Ravishankar S, et al. Wilson's disease: Cranial MRI observations and clinical correlation. Neuroradiology 2006;48(9):613-621. Epub 3 Jun 2006.

2. Kim TJ, Kim IO, Kim WS, et al. MR imaging of the brain in Wilson disease of childhood: Findings before and after treatment with clinical correlation. Am J Neuroradiol 2006;27(6):13731378.

3. Hitoshi S, Iwata M, Yoshikawa K. Mid-brain pathology of Wilson's disease: MRI analysis of three cases. J Neurol Neurosurg Psychiatry 1991;54:624-626.

4. Rutledge JN, Hilal SK, Silver AJ, et al. Study of movement disorders and brain iron by MR. Am J Roentgenol 1987;149:365-379. 5. Prashanth LK, Sinha S, Taly AB, et al. Do MRI features distinguish Wilson's disease from other early onset extrapyramidal disorders? An analysis of 100 cases. Mov Disord 2010;25(6):672678. [http://dx.doi.org/10.1002/mds.22689]

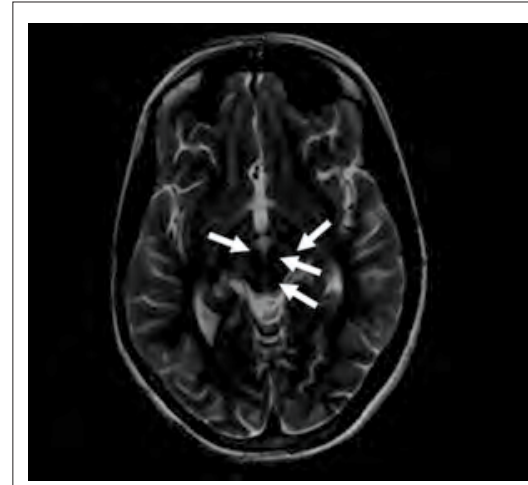

Fig. 1. Axial T2-weighted MRI of the brain at the level of the midbrain showing the characteristic 'face of the giant panda' sign, with normal red nuclei and substantia nigra (pars reticulata) against a background of hyperintensity in the tegmentum, as well as hypointensity of the superior colliculi.

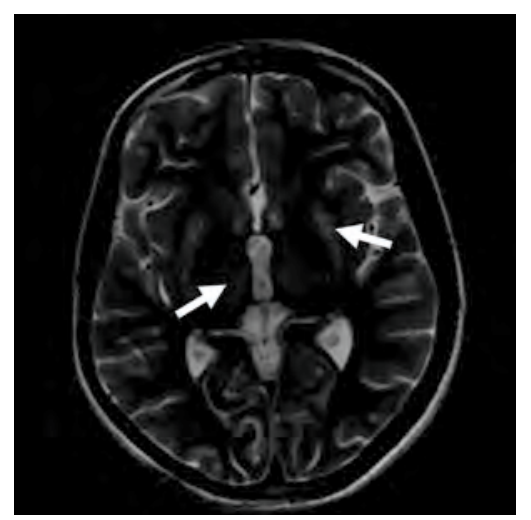

Fig. 2. Axial T2-weighted MRI of the brain at the level of the basal ganglia showing hyperintensity in the putamina and thalami (white arrows). 